East African Medical Journal Vol. 81 No. 7 July 2004

HYSTEROSCOPIC SURGERY AT THE AGA KHAN HOSPITAL, NAIROBI

R. B., Parkar, MBBS, MMed, Consultant Obstetrician, Gynaecologist and Laparoscopic Surgeon, P.O. Box 520, Sarit Centre, 00606, Nairobi, and N. G. Thagana,

MBChB, MMed, Consultant Obstetrician and Gynaecologist, P.O. Box 75163, Nairobi, Kenya

Request for reprints to: Dr. R. B. Parkar, P.O. Box 520, Sarit Centre 00606, Nairobi, Kenya

\title{
HYSTEROSCOPIC SURGERY AT THE AGA KHAN HOSPITAL, NAIROBI
}

\author{
R.B. PARKAR and N. G. THAGANA
}

\begin{abstract}
Objectives: To determine the experience, acceptability and outcome of hysteroscopic surgery at the Aga Khan Hospital, Nairobi.

Design: A retrospective study.

Setting: Aga Khan Hospital, Nairobi.

Patients: Between May 2000 and April, 2003, 463 cases of hysteroscopy were undertaken at the Aga Khan Hospital, Nairobi, of these 54 patients $(11.7 \%)$ underwent various hysteroscopic surgical procedures. A review of these cases and their outcomes are analysed and presented. Results: In a thirty six month period 463 cases of Hysteroscopy were done at the Aga Khan Hospital, Nairobi. Of these 54 cases had operative intervention $(11.7 \%)$. The case load of diagnostic hysteroscopy increased from 50 in the year 2000, to 206 and 159 respectively in the years 2001 and 2002 . By this time, some consultants were performing diagnostic hysteroscopies on their own. The surgical procedures performed included hysterscopic retrieval of " lost "IUCD's, and intrauterine bone spicules and hysteroscopic resection of submucous fibroids $(25.9 \%$ of the cases $)$, resection of endometrial polyps (25.9\%) hysteroscopic synechiolysis, (16.6\%) and endometrial resection or ablation (cauterisation) (7.4\%). All the patients were reviewed by the consultant pool after one week. Three cases of complications were encountered, two cases of uterine perforation and one case of fluid overload (TURP syndrome) following a resection of a submucous fibroid. Conclusion: Hysteroscopy and hysteroscopic surgery are the established gold standards for the management of intrauterine pathology. The procedures were well accepted amongst patients who had undergone these procedures.

Recommendations: In Kenya, gynaecologists still have to embrace change and undergo adequate and sustained training in the various aspects of hysteroscopy, so as to make the proecdures acceptable and accessable to more patients.
\end{abstract}

\section{INTRODUCTION}

Blind sampling with dilatation and curettage of the uterine cavity ( $\mathrm{D} \& \mathrm{C}$ ), was until recently a routine procedure in gynaecology. With the advent of advanced optics, safer distention techniques and adequately trained surgeons, hysteroscopy is now the recommended gold standard for the assessment and management of intrauterine pathology, and is regarded as a safe, acceptable and well tolerated procedure(1).

Pantalleoni in 1869 performed the first documented hysteroscopy in Bologna, Italy, using a cystoscope (1). Gimpelson et al. reported that hysteroscopy was far superior to the conventional dilatation and curettage in the evaluation of abnormal uterine bleeding(2).

Since the development of the appropriate technique of hysteroscopy in the early 70's(3,4), and advanced instrumentation over the last two decades, hysteroscopy is now regarded as the most reliable method for the diagnosis and treatment of intrauterine lesions and pathology(5).
Initially only minor therapeutic interventions could be performed, due to lack of adequate instrumentation. The original single flow hysteroscopes with only an inflow channel for the distention medium appeared to be a drawback(5). Blurring of vision, loss of adequate distention and caramalisation of instruments with dextran distention were added limitations(5). In 1978, Neuwirth introduced the hysteroscopic endoresection techniques which initiated a new era in transcervical hysteroscopic endosurgery(6). This technique opened up completely new avenues for minimally invasive and uterus preserving treatment of intrauterine disorders. Hallez further reported the use of the continuation flow hysteroresectoscope which significantly enhanced visualisation and safety(7).

Adequate uterine distention is an absolute prerequisite for modern hysteroscopic techniques(8). The media used should not be toxic with intravascular intravasation, should create adequate distention, of the uterine cavity and provide a permanent clear 
view(8). Various mediums have been tried and tested and include $\mathrm{CO}_{2}$ gas, high viscosity fluids (hyskon, dextran) and low viscosity fluids (sorbitol, glycine, mannitol). Saline can be used only for diagnostic procedures(8).

The major advantages of hysteroscopic surgery include, no laparotomy, no uterine wall scarring, shorter hospital stays, quicker recovery and good patient compliance(9). The role of hysteroscopy in the management of abnormal bleeding, infertility and uterine malformations, is now regarded as second to none(911). Miskry et al. recommended hysteroscopy as a first line procedure of choice in all women with persistent menstrual disturbances irrespective of their age or utrasound findings(12).

Large series of examinations and treatments under hysteroscopic control reveal comparatively few adverse reactions. In a review of 184,563 hysteroscopies by 101 hysteroscopists from various centres between 1986 and 1988, Lidemann reported a serious complication rate of $0.012 \%(13)$.

Although, hysteroscopy has been a well accepted modality worldwide, in Kenya, the acceptability of hysteroscopy as a first line procedure, is yet to be realised, as the awareness, commitment to change and adequate training become an acceptable reality.

\section{MATERIALS AND METHODS}

A retrospective case analysis was undertaken at the Aga Khan Hospital, Nairobi, of all cases of hysteroscopic surgery undertaken in the presence of the principle author. All cases whose records were incomplete or not retrieved, all diagnostic procedures, and cases performed at various other hospitals were not entered in the study.

The procedure of hysteroscopy was standard for all cases. An Olympus $5.5 \mathrm{~mm}$ CF hysteroscope sheath, $12^{\circ}$ or $30^{\circ}$ hysteroscope, with a working channel, and hystero resectoscope was used for all the surgical cases. Fluid distention was maintained with the aid of a $3 \mathrm{~L}$ Olympus pressure cuff, using purisol as the distention medium for all operative procedures. Normal saline was used for diagnostic procedures only. In addition at the Aga Khan Hospital, Nairobi, a standard Karl Storz tower with two Sony 21 inch monitors, a Tricam SL Pal Camera, a Xenon 300 light source, and an Autocon 350 high frequency diathermy unit were used.

The hysteroresectoscope, with a cutting loop electrode or a right angled knife electrode was used as a standard instrument, for all operative procedures. In a few suitable cases a rigid 3Fr scissor and grasper were used. In five cases the Vapourtrode was used, in the presence of a visiting overseas consultant.

All records were adequately maintained on a Toshiba satellite $4070 \mathrm{CDs}$ laptop computer. The results of 54 operative hysteroscopy cases were reviewed, presented and discussed herein.

\section{RESULTS}

Table 1

Total cases of hysteroscopy performed at the Aga Khan Hospital, Nairobi

\begin{tabular}{lccc}
\hline Year & $\begin{array}{c}\text { Diagnostic } \\
\text { Hysteroscopies }\end{array}$ & $\begin{array}{c}\text { Operative } \\
\text { Hysteroscopies }\end{array}$ & $\begin{array}{c}\text { Total } \\
\text { Hysteroscopies }\end{array}$ \\
\hline 2000 & 43 & 7 & 50 \\
(May to December) & & 16 & 206 \\
2001 & 190 & 20 & 159 \\
2002 & 139 & 11 & 48 \\
2003 & 37 & $54(11.7 \%)$ & 463 \\
(January to April ) & 409 &
\end{tabular}

Table 2

Indications for operative hysteroscopy

\begin{tabular}{ll}
\hline Primary infertility & 3 \\
Secondary infertility & $19(35.2 \%)$ \\
Menorrhagia & 14 \\
Leucorrhoea & 3 \\
Amenorrhoea & 9 \\
Postmenopausal bleeding & 6 \\
\hline
\end{tabular}

Table 3

Pathology seen on hysteroscopy

\begin{tabular}{ll}
\hline Submucous fibroid & \\
$\quad$ Single & $16(29.6 \%)$ \\
$\quad$ Multiple & 1 \\
Endometrial polyps & 15 \\
Bicornuate uterus & 1 \\
Foreign bodies (bone spicules) & 4 \\
Uterine Septum & 2 \\
Intrauterine adhesions (Ashermanns) & 7 \\
(Lost IUCD) & 2 \\
\hline
\end{tabular}

Table 4

Operative procedures performed

\begin{tabular}{|c|c|c|}
\hline \multicolumn{3}{|l|}{ Retrieval } \\
\hline & IUCD & 2 \\
\hline & Bone Spicules & 4 \\
\hline \multicolumn{3}{|l|}{ Resection } \\
\hline & Submucous fibroids & $14(25.9 \%)$ \\
\hline & Endometrial polyps & 14 \\
\hline & Endometrial ablation & 4 \\
\hline & Septum/adhesions & 9 \\
\hline \multicolumn{3}{|c|}{ Vapourisation } \\
\hline & Submucous fibroids & 3 \\
\hline & Endometrial polyps & 1 \\
\hline & Endometrium & 1 \\
\hline
\end{tabular}

(Minor operative procedures: 14, Major operative procedures $40-74 \%)$ 
Table 5

Complications encountered during operative hysteroscopy

\begin{tabular}{ll}
\hline Complications & No. of cases \\
\hline Uterine perforation & 2 \\
TURP syndrome (Fluid Overload ) & 1 \\
\hline
\end{tabular}

\section{DISCUSSION}

Hysteroscopy is the recommended method for the diagnosis and management of gynaecological complaints, including abnormal uterine bleeding, infertility, recurrent retained IUCD's and has replaced the traditional D and C (13). At the Aga Khan Hospital, Nairobi, diagnostic and operative hysteroscopies have been undertaken since may 2000 (Table 1). In the period reviewed, 409 diagnostic hysteroscopies and 54(11.7\%) operative procedures were undertaken. The indications for operative hysteroscopy are listed in Table $2.35 .2 \%$ of the cases were performed for secondary infertility (Table 2). Pathologies encountered included submucous fibroids in $29.6 \%$ (Table 3).

Stock and Kanbour reported in a series of patients who underwent $\mathrm{D}$ and $\mathrm{C}$ hysterectomy that in $60 \%$ of the specimens the endometrium had been sampled(14).

La Sala et al. documented a sensitivity of $100 \%$ and specificity of $86 \%$ for hysteroscopy(15). Decloedt and Fenton reported abnormal hysteroscopic appearances in $32 \%$ of the cases sampled. In our series $11.7 \%$ of the 463 patients had abnormal pathology and underwent hysteroscopic surgery.

De Blok and Wamsteker divided hysteroscopic surgery into minor and major procedures. Minor procedures induded resection of small polyps, selective poly, extraction of a non-embedded IUCD, synechiectomy of Ashermanns' Syndrome (Grade I, II), Ovabloc and related sterilizations, selective salpingography and hysteroscopic ZIFT and GIFT procedures.

Major endosurgical hysteroscopic procedures included resection of large endometrial polyps, retrieval of embedded or fragmented IUCD's, resection of large or multiple fibroids, resection of intrauterine septa and dense adhesions, and endometrial resection or ablation(17). In the series presented $26.0 \%$ of the procedures were minor while $74.0 \%$ major hysteroscopic procedures were undertaken (Table 4). At the Aga Khan Hospital, Nairobi, resection of submucous fibroids made up $25.9 \%$ of the operative hysteroscopic procedures undertaken and nine cases of intrauterine adhesions/ synaechia were encountered (Table 4).

Although intrauterine synechiae were first described by Fritsch, it was Ashermanns' publication in 1948 that outlined the clinical consequences of these adhesions(18). Hysteroscopic synechiolysis offers substantial advantages over the blind $\mathrm{D}$ and $\mathrm{C}$ procedures in patients presenting with hypomenorrhoea, amenorrhoea, repeated spontaneous abortions or a history of traumatic intrauterine manipulations or infections(18). In the seven patients with intrauterine adhesions, in the series treated by either sharp dissection or using a monopolar knife electrode, the commonest presentation was secondary amenorrhoea. Four of these patients were followed up, upto six months after the procedure and reported regular menses (Table 2).

Uterine fibroids with intracavitary protrusion may present with menorrhagia, metroraghia, dysmenorrhoea, infertility, early foetal loss, premature labour. The intracavitary extention of uterine fibroids (submucous fibroids) can be established by hysteroscopy(19). In our series, secondary infertility, menorrhagia and in one patient leucorrhoea were the presenting symptoms. Although the symptoms improved in majority of patients, conception rates have not as yet been established. It was Neuwirth in 1976 who opened new possibilities for the treatment of submucous fibroids, who reported success rates of $90 \%-94 \%$ in the subjective treatment of menorrhagia(6). Hallez introduced the continuous flow hysteroresectoscope and the use of electrolyte free low viscosity distention fluid, in 1987, and this technique is now the most accepted method of hysteroscopic resection of submucous fibroids(7). In our series the loop electrode with monopolar cutting current was the commonest mode of resection. In two patients however, with small pedunculated submucous fibroids the stalk was resected using scissors.

In a group of 94 patients, Derman et al. in 1991, reported that $83.9 \%$ of patients undergoing hysteroscopic resection of submucous fibroids for menorrhagia, required no further surgical intervention. In this group, 21 patients conceived and delivered 18 living infants(20). Feng et al. noted a $94.95 \%$ improvement in the menstrual status of 99 patients undergoing transcervical resection of submucous fibroids(21). In the series presented, 17 cases of submucous fibroids were handled successfully. One patient with four large submucous fibroids underwent a second hysteroscopic resection six weeks following the first. The European Society of Hysteroscopy introduced a classification for the intramural and intracavitary extent of submucous fibroids. The type $\mathrm{O}$ submucous fibroid is pedunculated and has no intramural extension.

In type I submucous fibroids the intramural extention is less than $50 \%$ and in type II fibroids the intramural component is more than 50\%(22). In the series presented, of the 17 cases of submucous fibroids encountered, nine were type $\mathrm{O}(52.9 \%)$, seven were type I ( $41.2 \%)$, and one was a type II. Of the cases 16 were single fibroids of varying sizes and one case had four submucous fibroids.

The results of hysteroscopic resection of submucous fibroids have been published in numerous articles and confirm the effectiveness of this technique. Excessive bleeding caused by intrauterine fibroids is normalised 
in upto $90 \%$ of the cases(22). Loffer published the results of 53 cases of hysteroscopic management of submucous fibroids. The long term results based on their series were that in 45 patients followed up for more than 12 months, $93 \%$ of the patients experienced a significant reduction in the excessive bleeding and only $9 \%$ of the said patients underwent a subsequent hysterectomy(23). Two of these patients had subsequent endoresection, while two patients underwent a non hysteroscopic myomectomy. Seven of the 12 infertility paffents (58\%) delivered subsequently(23).

Dermann et al. summarised the long term follow up of patients undergoing resectoscopic management of submucous fibroids(20). Of the 94 patients managed between 1973 and 1988, 83.9\% did not require any further surgical management(20).

Complications of hysteroscopy can be divided into complications of surgery, and complications resulting from the distention medium. During resection, uterine wall perforation may occur causing serious intestinal or vascular injuries. More importantly a more fatal complication may occur as a result of fluid overload, pulmonary oedema, electrolyte imbalance and fluid intravasation $(22,23)$. Hallez et al. reported one case of uterine perforation during 61 endoresections, which was recognised and repaired immediately by laparotomy(7). Brooks et al. mentioned one perforation of the uterus during extraction of the resected pieces of a fibroid, and one case of febrile endometritis following 92 endoresections(19). Of 55 endosurgical procedures using a resectoscope, Loffer reported no major complications. Two patients had more than $1000 \mathrm{mls}$ of fluid retention with transient hyponatraemia and another had uterine perforation during retrieval of the intrauterine resected chips(23). Derman et al. in treating 94 women, noted that four required blood transfusion, two underwent laparotomy, and one had a uterine perforation. One patient developed pulmonary oedema and hyponatraemia, caused by fluid overload(20). In our series two cases of perforation were encountered, which were managed by laparoscopic repair of the uterine defect. In one case of fluid overload encountered, ICU admission was warranted. She was managed with intravenous saline infusion and intravenous lasix. The patient recovered well.

Miskry et al. recommended hysteroscopy as a first line procedure of choice in all women with persistent menstrual disturbances, irrespective of the ultrasound findings or their age(12). Hysteroscopic high frequency electro endosurgery is an elegant and gentle technique and now the method of choice for the management of intrauterine pathology. Motashaw and Dave reported that the complications of hysteroscopy are related to the experience of the surgeons, their knowledge of the anatomy and their familiarity with instruments. No surgeon is immune from encountering complications, most importantly the complications, should be recognised and treated promptly(24).

\section{ACKNOWLEDGEMENTS}

To all the colleagues who provided the various cases for this study, and to the administration and staff of the Aga Khan Hospital, Nairobi, and the patients for their availability in making this publication a reality.

\section{REFERENCES}

1. Alexopoulos E.D., Fay T. N., and Simonis, C.D., A review of 2581 outpatient diagnostic hysteroscopies in the management of abnormal uterine bleeding. Gynaecol. Endo. 1999; 8:105-110.

2. Gimpelson R., and Rappold H. A comparative study between panoramic hysteroscopy with directed biopsies and dilatation and curettage. Amer. Obstet Gynaeco. 1988; 158:489-492.

3. Edstrom F., and Fernstrom, I., The diagnostic possibilities of a modified hysteroscopic technique. Acta Obstet. Gynea. Scandindivica. 1970; 49:327-330.

4. De Cherney A., and Polan M.L. Hysteroscopic management of intrauterine lesions and intractable uterine bleeding. Obstet. Gynae. 1983; 61:392-394.

5. Wamsteker K., De Blok S., and Emanuel M.H. Instrumentation for transcervical hysteroscopic endosurgery. (Editorial) Gynae. Endo. 1992; 1:59-67.

6. Neuwirth, R.S., A new technique for and additional experience with hysteroscopic resection of submucous fibroids. Amer. Obstet. Gynaecol. 1978; 131:91-94.

7. Hallez, P. Netter A., and Cartier R., Methodical intrauterine resection. Amer. J. Obstet. Gynae. 1987; 256:1080-1084.

8. Wamsteker K. and De Blok, S., Diagnostic hysteroscopy: technique and documention. In: Sutton C., Diamond M.P., editors, Endoscopic Surgery for Gynae. London, 1993; WB Saunders Comp Ltd.

9. Dimitrios K., and Zourlas H., Transcervical division of uterine septa. Obstet. Gynecol. Surv. 1990; 45:165.

10. Motashaw, N.D. and Dave, S. Diagnostic and therapeutic hysteroscopy in the management of abnormal uterine bleeding. Repro. Med. 1990; 35:616-620.

11. Mancaglia, L. Colafranceschi, M. and Gordon, A.G., Is hysteroscopy of value in the investigation of female infertility? Acta. Eur. Fertil. 1988; 19:239-242.

12. Miskry, T., Rauch, M., and Magos, A. Hysteroscopy in women aged 30 years or less. Gynaecol. Endo. 2000; 9:315-317.

13. Lidemann, H.J., Historical aspects of hysteroscopy. Fertil. Steril. 1973; 24:230-243.

14. Stock, R., and Kanbour, A. Pre hysterectomy curettage Obstet. Gynae. 1975; 45:537-541.

15. La Sala G.B., Sacchei, F. and Dessanti, L., Panoramic diagnostic micro hysteroscopy: analysis of results obtained from 976 out patients. Acta. Obste. et Gynea. Scandinavica. 1994; 141:65-67.

16. Decloedt, F., and Fenton, D.W. Outpatient hysteroscopy: indications and hysteroscopic findings in pre and postmenopausal patients. Gynaecol. Endo. 1999; 8:137-141.

17. de Blok, S. and Wamsteker, K. Hysteroscopic surgery, possibilities, limitations and future. Rep Med Rev, 1995; 4:101-113.

18. Shenker, G.A. and Margalioth, E.J. Intrauterine adhesions: an updated appraisal, Fertil. Steril. 1982; 37:593-599.

19. Brooks, P.G., Loffer, F.D. and Serdan, S.P. Resectoscopic removal of symptomatic intrauterine lesions. J. Reprod. Med. 1989; 34:435-437. 
20. Derman, S.G., Rehnstrom, J. and Neuwirth, R.S. The long term effectiveness of hysteroscopic treatment of menorrhagia and leiomyomas. Obstet. Gynecol. 1991; 77:591-594.

21. Feng, Z.G., Shi, Y.P., and Liu S.P., Hysteroscopic resection of submucous fibroids clinical analysis of 99 cases. Gynaecol. Endo. 2002; 11:127-130.

22 Wamsteker, K., Emanuel, M.H., and de Kruif, J.H., Transcervical hysteroscopic resection of submucous fibroids for abnormal uterine bleeding: Results regarding the degree of intramural extension Obstet. and Gynae. 1993; 82:5:736-740.

23. Loffer, F.D. Removal of large symptomatic intrauterine growths by the hysteroscopic resectoscope. Obstet. Gynaecol. 1990; 76:836-840.

24. Motashaw, N.D., and Dave, S. Complications of hysteroscopy (Commentary). Gynaecol. Endo. 2001; 10:203-210. 